\title{
Evaluating the sustainability of a smart technology application to mobile health care: the FGM-ACO-FWA approach
}

\author{
Tin-Chih Toly Chen ${ }^{1}$ (D)
}

Received: 28 January 2019 / Accepted: 21 August 2019 / Published online: 31 August 2019

(C) The Author(s) 2019

\begin{abstract}
Smart technologies present numerous opportunities for enhancing mobile health care. However, existing applications of smart technologies to mobile health care face several difficulties. As a result, whether a smart technology application to mobile health care will be sustainable is questionable. To address this issue, the fuzzy geometric mean (FGM)- $\alpha$-cut operations (ACO)-fuzzy weighted average (FWA) approach is proposed in this study. In the proposed methodology, at first FGM is applied to aggregate multiple experts' opinions on the relative importance of a critical factor. Then, ACO is applied to derive the absolute fuzzy importance level of the critical factor. At last, FWA is applied to assess the sustainability of the smart technology application to mobile health care. The proposed methodology has been applied to assess the sustainability of thirteen smart technology applications to mobile health care. According to the experimental results, the most and least sustainable smart technology applications to mobile health care were smart mobile service and smart clothes, respectively. In addition, the ranking result using the proposed methodology was somewhat different from those using existing methods based on approximation.
\end{abstract}

Keywords Mobile $\cdot$ Health care $\cdot$ Smart technology $\cdot$ Fuzzy geometric mean $\cdot \alpha$-Cut operations $\cdot$ Fuzzy weighted average $\cdot$ Sustainability

\section{Introduction}

Smart technologies are technologies that use electronic devices or systems that can be connected to the Internet, used interactively, and are to some extent intelligent [19]. Smart technologies have been applied to provide superior health care services to mobile users [22, 27]. For example, mobile guides such as Google Maps direct users after detecting their locations through the global positioning system (GPS) to enable users to reach destinations as soon as possible, thereby increasing their comfort and reducing their tiredness [11, 13]. Smart watches have full mobile phone capability and are equipped with many sensors, such as thermometers and heart rate monitors, to monitor and help maintain users' physical conditions, which are then conveyed to the backend server for further diagnosis [35]. Apps

Tin-Chih Toly Chen

tolychen@ms37.hinet.net

1 Department of Industrial Engineering and Management, National Chiao Tung University, 1001 University Road, Hsinchu, Taiwan have been developed to guide a mobile patient to the nearest clinic that provides the required treatment and satisfies the patient's preferences [12]. In a smart hospital, wireless sensors are attached to mobile patients to collect real-time information such as their pulses and oxygen saturation readings [17]. The location of a patient with a smart phone can be detected by comparing the WiFi signal strengths in different zones [43].

In summary, smart technologies have the following benefits:

1. Smart technologies can be applied to provide health care assistance to mobile users in both indoor and outdoor environments.

2. Some smart technologies can overcome the limitations of existing technologies.

3. Smart technologies are more effective in guiding a user to lead healthy lifestyles than in providing health care services or health care resource information to the user.

However, existing applications of smart technologies to mobile health care face several difficulties. First, some smart 
technologies are obtrusive, which discourages users, particularly mobile users, from applying such smart technologies. For example, monitoring autonomic nervous system activities requires a large wrist device to be worn by a user, which is obviously obtrusive [4]. Smart glasses are heavy and striking to put on despite their advantages in providing real-time information and instructions. Smart clothes are uncomfortable to wear even if they can detect the most real-time health conditions. In addition, it is still a challenging task to aggregate the collected heterogeneous data from multiple sources [2]. As a result, the applications of some smart technologies to mobile health care may not sustain. To address this issue, the fuzzy geometric mean (FGM)- $\alpha$-cut operations (ACO)-fuzzy weighted average (FWA) approach is proposed in this study to evaluate the sustainability of a smart technology application to mobile health care. The motives for this study include the following:

1. A systematic way for evaluating the sustainability of a smart technology application to mobile health care is still lacking [31]. In the literature, only [18] modeled the improvement in the successful recommendation rate of a clinic recommendation system with a learning process, so as to evaluate the sustainability of the clinic recommendation system.

2. The critical factors for ensuring the sustainability of a smart technology application to mobile health are usually subjective [15], which can be properly handled using FAHP.

3. Existing FAHP methods are usually based on approximation $[1,6]$. However, the precise weights of critical factors can provide more information than approximated weights.

To address these issues, the following treatments are taken in this study:

1. Factors critical to the sustainability of smart technology applications to mobile health care are discussed.

2. Multiple experts compare the relative importance levels of critical factors using a FAHP method that is composed of FGM and ACO.

3. FGM is applied to aggregate experts' opinions. In contrast, most of the past studies (e.g., [24, 29, 33]) applied fuzzy arithmetic mean (FAM), which led to an inappropriate aggregation result if the pairwise comparison results by two experts were contrary to each other.

4. ACO is applied to derive the fuzzy eigenvalue and eigenvector. The fuzzy eigenvector is normalized to derive the absolute fuzzy importance level of each critical factor. In contrast, in most of the past studies, FGM and fuzzy extent analysis (FEA) were applied to fulfill the same purpose $[24,40]$. However, FGM and FEA are approxi- mation techniques, and may overestimate or underestimate the absolute fuzzy importance level of a critical factor.

5. From the FAHP results, FWA is applied to assess the sustainability of a smart technology application to mobile health care.

The novelty and originality of the proposed methodology reside in the following:

1. The sustainability of smart technology applications to mobile health care has rarely been discussed in the past. Most of the past studies on similar topics were focused on the acceptance (or adoption) of a limited number of smart devices such as smart phone, smart home, and smart watch. For example, Chen et al. [10] applied innovation diffusion theory (IDT), technology acceptance model (TAM), and self-efficacy theory to find out the factors affecting the adoption of smart phones in the logistics industry. For the same purpose, Chen et al. [9] applied TAM and self-efficacy theory in the delivery service industry. Gaul and Ziefle [23] applied TAM to find out factors affecting the acceptance of a smart medical stent for monitoring health status in a smart home. After a TAM analysis, Kim and Shin [28] concluded that the determinants of smart watch adoption included affective quality, relative advantage, mobility, availability, and subcultural appeal. However, the topic discussed in this study is not limited to specific smart devices. In addition, sustainability, unlike technology acceptance, is a long-term concept.

2. In addition, most of the past studies on similar topics were based on questionnaires [10,23], while the proposed methodology is based on experts' pairwise comparison results.

3. Although FGM, ACO, and FWA have been applied in the past FAHP studies [5, 39], the combination of FGM, $\mathrm{ACO}$, and FWA has rarely been investigated. This combination ensures a precise deriving of the absolute fuzzy importance of a critical factor.

The remainder of this paper is organized as follows. Section 2 is dedicated to a literature review. Section 3 details the FGM-ACO-FWA approach for assessing the sustainability of a smart technology application to mobile health care. A case study has been conducted to illustrate the applicability of the FGM-ACO-FWA approach, as described in Sect. 4. Some existing methods are also applied to the case for a comparison. Finally, Sect. 5 presents concluding remarks and provides some directions for future research. 


\section{Literature review}

\section{Classification of smart technologies}

Smart technologies and systems can be classified into four categories [44]:

1. Wirelessly connected smart systems, such as Internet of things, smart TV, smart baby monitor, smart phone, smart watch, smart wallet, smart motion sensor, smart smoke alarm, smart glasses, spectacles and contact lenses, smart body analyzer, smart thermostat, and smart city.

2. Interactive smart systems, such as smart table, smart board, smart burglar alarm, smart fridge, smart cooker, smart washing machine, smart light bulb, smart meter, and smart plug.

3. Predictive smart systems, such as smart hard drive, smart connected vehicle, smart building, smart traffic light, smart surveillance camera, smart toilet, smart wig, smart farm, smart grid, and smart shelf.

4. Intelligent smart systems, such as smart $\mathrm{WiFi}$, smart mobile service, smart restart, smart defense, smart pajama, smart vacuum cleaner, smart travel card, smart wheelchair, and sleep-monitoring system.

According to Demirkan [21], the three types of smart technologies that can be applied to mobile health care are biosensor, wearable device, and intelligent software agent. According to Chen and Chiu (2016), smart technologies suitable for supporting mobile health care include smart watch, smart motion sensor, smart body analyzer, smart connected vehicle, smart wig, smart mobile service, smart defense technology, and smart wheelchair. Obviously, not all of existing smart technologies are applicable in a mobile environment.

\section{Applications of smart technologies to mobile health care}

Smart phones are the handiest and prevalent smart technology for mobile health care. In addition to cameras and a global positioning system (GPS) receiver, a smart phone is equipped with a variety of sensors such as accelerometer, proximity sensor, compass (or magnetometer), gyroscope, barometer, photometer, and thermometer. These sensors are either hardware or software [34], and can be divided into motion sensors, environmental sensors, and position sensors [3]. There have been sufficient evidences supporting the effectiveness of smart phone applications to mobile health care. For example, messaging services help to prevent the risk factors for cardiovascular diseases, cancer, diabetes, and chronic respiratory diseases, and improve the perceived self-care $[22,47]$. However, simple text messaging may not lower the mortality rate [22]. The trend is towards the usage of apps and online social networks. Most of existing apps are intended for use by consumers or patients. Only few of them are targeted at health care professionals [36]. In addition, some health care professionals are reluctant to use mobile health care apps [46].

A wrist-worn device is usually equipped with sensors such as compass, GPS, heart rate monitor, gyroscope, accelerometer, barometer, vibration motor, and photoplethysmography sensor. These devices can be applied to track the energy expenditure (EE) (i.e., the calories burned), steps taken, distance traveled, and heart rate (HR) [42]. Shcherbina et al. [38] assessed the accuracy of seven commercially available wrist-worn devices in estimating HR and EE. The results were compared with those measured with continuous telemetry and indirect calorimetry. According to the experimental results, the error rate was the lowest when a user was cycling, and was the highest when the user was walking, which seemed to be a big problem since walking is the most common motion in our daily lives. In addition, a higher error rate was associated with male, darker skin color, and higher body mass index. On average, the error rates of estimating HR and EE using wrist-worn devices were $5 \%$ and up to $20 \%$, respectively. The most representative wrist-worn device is smart watch. The combination of a smart watch (with an accelerometer) and a smart phone (with a vibration feedback) has been demonstrated to be a convenient and effective means for developing user-system interaction based on gesture recognition [7, 32]. For this purpose, an app based on existing application program interfaces (APIs) must be installed on a smart phone in advance to preprocess the collected data, perform client-side reasoning, transmit the collected data to the backend server, and receive a recommendation message. Porzi et al. [32] observed that an accelerometer on a smart watch usually samples at a rate much higher than the rate that can be handled by the APIs on a smart phone, which makes the preprocessing step essential. The compatibility of a smart watch with various types of apps is, therefore, critical [42].

A smart motion sensor is an essential device for a smart home for detecting the motion of a resident and comparing the detection result with normal motions or the resident's routine. Sixsmith and Johnson [41] used a smart motion sensor to detect the possible falls of an elderly person. Isken et al. [26] demonstrated the feasibility of using a sensor network for collecting the data of patient flows through health care clinics. However, according to the survey conducted by Demiris et al. [20], a smart motion sensor was more valuable for elderly people in detecting intruders, which 
reduced anxiety. Moreover, compared with other smart home devices, smart motion sensors were less intrusive.

However, a major barrier hampering existing applications of smart technologies is the related laws that restrict the possibility of distant medical care [25].

\section{The proposed methodology}

\section{FGM for aggregating multiple experts' evaluation results}

The proposed methodology uses a group of $K$ experts. First, each expert performs a pairwise comparison of the relative importance levels of factors critical to the sustainability of a smart technology application. The results are put in a fuzzy pairwise comparison matrix:

$\tilde{\mathbf{A}}_{n \times n}(k)=\left[\tilde{a}_{i j}(k)\right] ; \quad i, j=1 \sim n ; \quad k=1 \sim K$,

where

$\tilde{a}_{i j}(k)=\left\{\begin{array}{ll}1 & \text { if } \quad i=j \\ \frac{1}{\tilde{a}_{j i}(k)} & \text { otherwise }\end{array} ; \quad i, j=1 \sim n ; \quad k=1 \sim K\right.$

$\tilde{a}_{i j}(k)$ is the fuzzy pairwise comparison result by expert $k$ for the relative importance of criterion $i$ over criterion $j$. Equation (2) is the reciprocal requirement for a fuzzy judgement matrix. The following linguistic terms, all in triangular fuzzy numbers (TFNs), are provided for the experts to choose from (Stefanini et al. 2008):

As important as: $\tilde{a}_{i j}(k)=(1,1,3)$

Slightly more important than: $\tilde{a}_{i j}(k)=(1,3,5)$

Considerably more important than: $\tilde{a}_{i j}(k)=(3,5,7)$

Extremely more important than: $\tilde{a}_{i j}(k)=(5,7,9)$

Absolutely more important than: $\tilde{a}_{i j}(k)=(7,9,9)$

These TFNs can be tailored to a specific expert group [14]. It is also possible to choose a value between two neighboring linguistic terms. $\tilde{a}_{i j}(k)$ is a positive comparison if $\tilde{a}_{i j}(k) \geq 1$. To aggregate the results by all experts, FGM is applied:

$\tilde{a}_{i j}=\sqrt[K]{\prod_{k=1}^{K} \tilde{a}_{i j}(k)} ; \quad k=1 \sim K$.

Theorem 1 Equation (3) meets the reciprocal requirement for a pairwise comparison matrix.
Proof Substituting Eqs. (2) into (3) gives

$$
\begin{aligned}
\tilde{a}_{i j} & =\sqrt[K]{\prod_{k=1}^{K} \tilde{a}_{i j}(k)} \\
& =\sqrt[K]{\prod_{k=1}^{K} \frac{1}{\tilde{a}_{j i}(k)}} ; \quad k=1 \sim K \\
& =\sqrt[K]{\frac{1}{\prod_{k=1}^{K} \tilde{a}_{j i}(k)}} \\
& =\frac{1}{\sqrt[k]{\prod_{k=1}^{K} \tilde{a}_{j i}(k)}} \\
& =\frac{1}{\tilde{a}_{j i}}
\end{aligned}
$$

Theorem 1 is proved.

It is well known that theoretically the product of $K$ TFNs is not a TFN anymore. Nevertheless, after taking the $K$-th root, the FGM of $K$ TFNs can be approximated with a TFN.

Subsequently, the fuzzy eigenvalue and eigenvector of $\tilde{\mathbf{A}}$, indicated, respectively, with $\tilde{\lambda}$ and $\tilde{\mathbf{x}}$, are derived by solving the following two equations [37]:

$\operatorname{det}(\tilde{\mathbf{A}}(-) \tilde{\lambda} \mathbf{I})=0$

and

$(\tilde{\mathbf{A}}(-) \tilde{\lambda} \mathbf{I})(\times) \tilde{\mathbf{x}}=0$,

where $(-)$ and $(X)$ denote fuzzy subtraction and multiplication, respectively. However, the two equations are not easy to solve. For this reason, most of the past studies applied approximation techniques such as FGM (not the FGM for aggregation) [48] and FEA. In contrast, in the proposed methodology, ACO is applied to derive the values of $\tilde{\lambda}$ and $\tilde{\mathbf{x}}$ precisely, which will be described in the next section.

Based on the values of $\tilde{\lambda}$ and $\tilde{\mathbf{x}}$, the fuzzy maximal eigenvalue and the fuzzy weight of each critical factor are derived, respectively, as

$\tilde{\lambda}_{\max }=\max \tilde{\lambda}$,

$\tilde{w}_{i}=\frac{\tilde{x}_{i}}{\sum_{j=1}^{n} \tilde{x}_{j}}$.

Based on $\tilde{\lambda}_{\text {max }}$, the consistency among the fuzzy pairwise comparison results is evaluated as

Consistency index $\widetilde{C . I} .=\frac{\tilde{\lambda}_{\max }-n}{n-1}$, 
Consistency ratio $\widetilde{C . R} .=\frac{\widetilde{C . I}}{\text { R.I. }}$,

where R.I. is the random index [37]. The fuzzy pairwise comparison results are inconsistent if $\widetilde{C . I} .>0.1 \sim 0.3$ or $\widetilde{C . R} .>0.1 \sim 0.3$, depending on the matrix size ([37]; Wedley 1993; Business Performance Management Singapore, 2013).

\section{ACO for deriving the fuzzy eigenvalue and eigenvector}

Complicated operations on fuzzy variables can be performed via their $\alpha$ cuts. For this reason, fuzzy parameters and variables in Eqs. (5) and (6) are replaced with their $\alpha$ cuts, which leads to

$\operatorname{det}(\mathbf{A}(\alpha)-\lambda(\alpha) \mathbf{I})=0$,

$(\mathbf{A}(\alpha)-\lambda(\alpha) \mathbf{I}) \mathbf{x}(\alpha)=0$,

where each $\alpha$ cut is an interval:

$a_{i j}(\alpha)=\left[a_{i j}^{L}(\alpha), a_{i j}^{R}(\alpha)\right]$,

$\lambda(\alpha)=\left[\lambda^{L}(\alpha), \lambda^{R}(\alpha)\right]$,

$\mathbf{x}(\alpha)=\left[\mathbf{x}^{\mathbf{L}}(\alpha), \mathbf{x}^{\mathbf{R}}(\alpha)\right]$,

where $L$ and $R$ indicate the left and right $\alpha$ cuts of a fuzzy variable, respectively. If $\alpha$ takes 11 possible values $(0,0.1$, $\ldots, 1)$, Eqs. (11) and (12) need to be solved $10 \cdot 2^{\mathrm{C}_{2}^{n}}+1$ times to derive the membership functions of fuzzy eigenvalue and eigenvector. In the past studies like Csutora and Buckley (2001), only the results when $\alpha=0$ or 1 were derived to simplify the computation.

Substituting Eqs. (15) into (8) gives

$$
\begin{aligned}
w_{i}(\alpha) & =\left[w_{i}^{L}(\alpha), w_{i}^{R}(\alpha)\right] \\
& =\left[\min _{*} \frac{x_{i}^{*}(\alpha)}{\sum_{j=1}^{n} x_{j}^{*}(\alpha)}, \max _{*} \frac{x_{i}^{*}(\alpha)}{\sum_{j=1}^{n} x_{j}^{*}(\alpha)}\right],
\end{aligned}
$$

which is a normalization process. The process of deriving fuzzy eigenvalue and eigenvector involves many fuzzy multiplications. Therefore, the fuzzy maximal eigenvalue and weights may not be well approximated with TFNs.

\section{FWA for evaluating the sustainability of a smart technology application to mobile health care}

According to the derived fuzzy weights, FWA is applied to evaluate the sustainability of a smart technology application to mobile health care as follows:

$\tilde{S}_{k}=\frac{\sum_{i=1}^{n} \tilde{w}_{i}(\times) \tilde{p}_{k i}}{\sum_{i=1}^{n} \tilde{w}_{i}}$

where $\tilde{S}_{k}$ is the sustainability of the $k$-th smart technology application to mobile health care; $\tilde{p}_{k i}$ is the performance of the $k$-th smart technology application along the $i$-th dimension. Applying ACO to Eq. (17) gives

$S_{k}^{L}(\alpha)=\min _{*, \#}\left(\frac{\sum_{i=1}^{n} w_{i}^{*}(\alpha) p_{k i}^{\#}(\alpha)}{\sum_{i=1}^{n} w_{i}^{*}(\alpha)}\right)$,

$S_{k}^{R}(\alpha)=\max _{*, \#}\left(\frac{\sum_{i=1}^{n} w_{i}^{*}(\alpha) p_{k i}^{\#}(\alpha)}{\sum_{i=1}^{n} w_{i}^{*}(\alpha)}\right)$,

where the two symbols * and \# can be $R$ or $L$, representing a right or left $\alpha$ cut. Then, the center-of-gravity (COG) formula [45] is applied to defuzzify $\tilde{S}_{k}$ to generate a crisp (or representative) value:

$D\left(\tilde{S}_{k}\right)=\frac{\sum_{\alpha=0}^{1} \alpha\left(\frac{S_{k}^{L}(\alpha)+S_{k}^{R}(\alpha)}{2}\right)}{\sum_{\alpha=0}^{1} \alpha}$,

which is basically the weighted average of the $\alpha$ cuts of $\tilde{S}_{k}$.

\section{A case study}

According to the results of the literature review, the following five factors seem to be more critical to the applicability of smart technologies to mobile health care:

Table 1 The results of pairwise comparisons

\begin{tabular}{llllll}
\hline & CF1 & CF2 & CF3 & CF4 & CF5 \\
\hline CF1 & 1 & $(3,5,7),(5,7,9),(5,7,9)$ & $(7,9,9),(3,5,7),(5,7,9)$ & $(5,7,9),(7,9,9),(5,7,9)$ & $(3,5,7),(3,5,7),(1,3,5)$ \\
CF2 & - & 1 & $(1,3,5),(2,4,6),(1,3,5)$ & $(3,5,7),(1,3,5),(2,4,6)$ & - \\
CF3 & - & - & 1 & - & $(3,5,7),(2,4,6),(3,5,7)$ \\
CF4 & - & - & $(1,1,3),(1,3,5),(1,1,3)$ & 1 & $(5,7,9),(3,5,7),(4,6,8)$ \\
CF5 & - & $(1,3,5),(3,5,7),(2,4,6)$ & - & - & 1 \\
\hline
\end{tabular}


Table 2 The aggregation results

\begin{tabular}{llllll}
\hline & CF1 & CF2 & CF3 & CF4 & CF5 \\
\hline CF1 & 1 & $(4.22,6.26,8.28)$ & $(4.72,6.80,8.28)$ & $(5.59,7.61,9.00)$ & $(2.08,4.22,6.26)$ \\
CF2 & - & 1 & $(1.06,3.30,5.31)$ & $(1.82,3.91,5.94)$ & - \\
CF3 & - & - & 1 & - & $(2.62,4.64,6.65)$ \\
CF4 & - & - & $(1.00,1.44,3.56)$ & 1 & $(3.91,5.94,7.96)$ \\
CF5 & - & $(1.82,3.91,5.94)$ & - & - & 1 \\
\hline
\end{tabular}

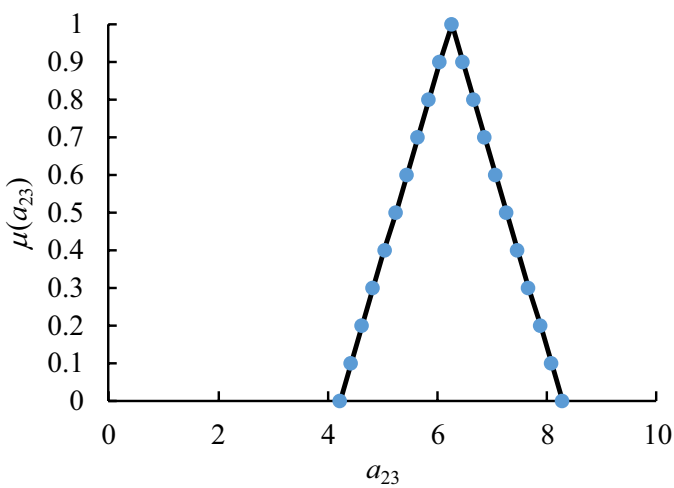

Fig. 1 Fitting the FGM result with a TFN

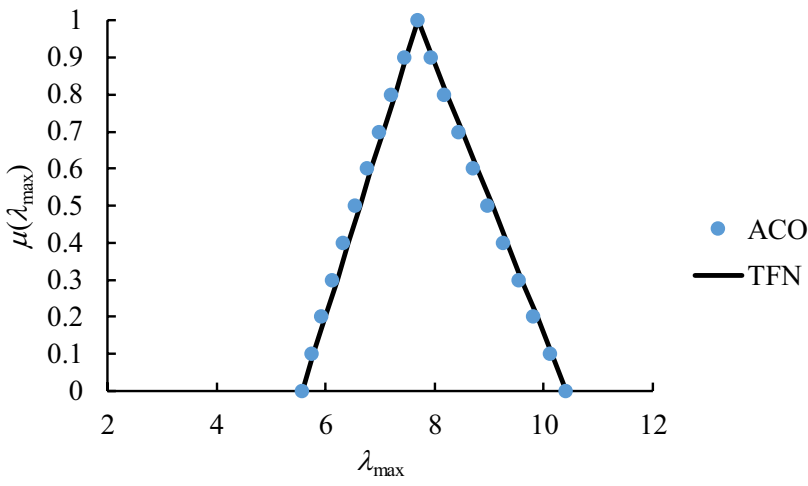

Fig. 2 The derived fuzzy maximal eigenvalue

1. CF1: unobtrusiveness $[7,20,32]$,

2. CF2: supporting online social networking [22, 47],

3. CF3: the relaxation of the related medical laws $[25,46]$,

4. CF4: the size of the health care market $[36,46]$, and

5. CF5: the correct identification of a user's need and situation $[7,32,38]$.

To determine the importance levels of critical factors, the proposed methodology was applied.

First, a group of three experts from medical and information science fields was formed. Each expert performed a pairwise comparison of the relative importance levels of critical factors. The results are summarized in Table 1.

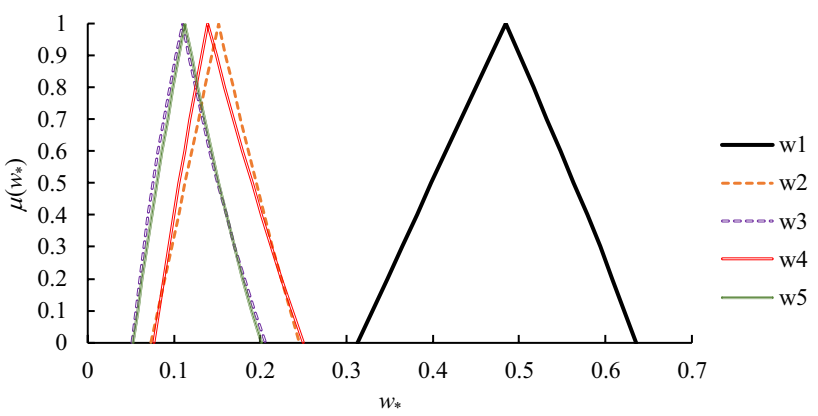

Fig. 3 The derived fuzzy weights
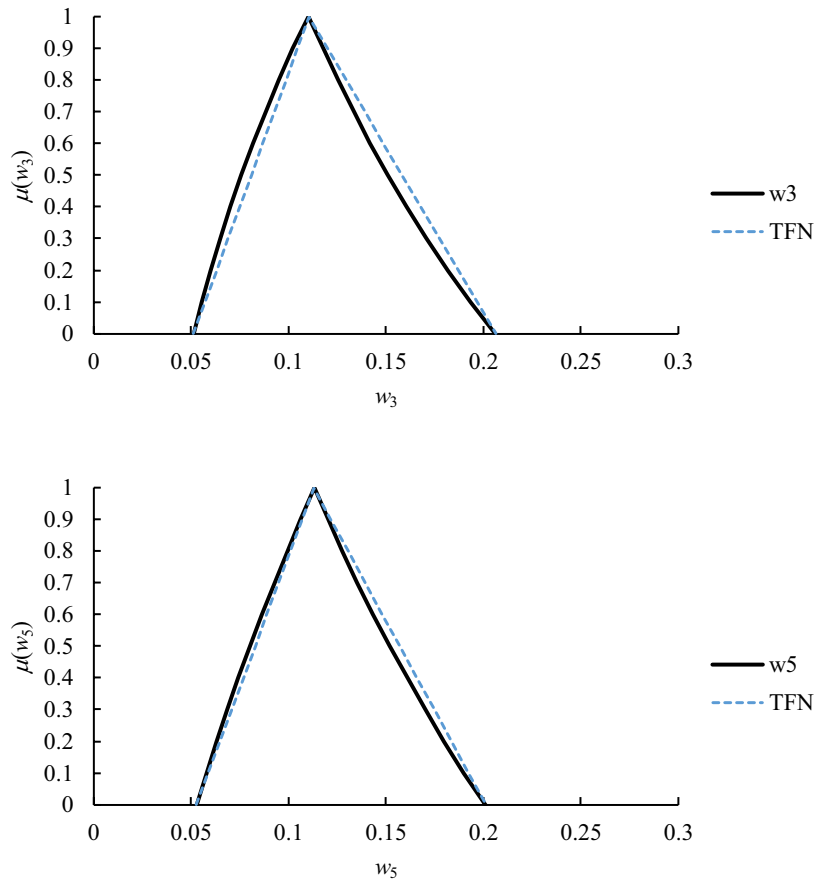

Fig. 4 Approximating $\tilde{w}_{3}$ and $\tilde{w}_{5}$ with TFNs

To aggregate the pairwise comparison results by experts, FGM was applied. The results are shown in Table 2. The FGM result was approximated with a TFN. It can be seen from Fig. 1 that a TFN fitted the FGM result well in the experiment.

Subsequently, ACO was applied to derive the fuzzy maximal eigenvalue and weights from the aggregation 
Table 3 The performances of thirteen smart technologies along the four dimensions

\begin{tabular}{llllll}
\hline $\begin{array}{l}\text { Requirement smart technol- } \\
\text { ogy }\end{array}$ & CF1 (unobtrusiveness) & $\begin{array}{l}\text { CF2 (online } \\
\text { social network- } \\
\text { ing) }\end{array}$ & CF3 (relaxation of law) & CF4 (market size) & CF5 (correct identification) \\
\hline Smart clothes & $(0,1,2)$ & $(0,1,2)$ & $(0,1,2)$ & $(0,1,2)$ & $(3.17,4.17,4.5)$ \\
Smart glasses & $(1,1.33,2.33)$ & $(3.33,4.33,5)$ & $(3.17,4.17,4.5)$ & $(0,1,2)$ & $(3.17,4.17,4.5)$ \\
Smart watch & $(3.67,4.67,5)$ & $(3.17,4.17,4.5)$ & $(3.67,4.67,5)$ & $(3.17,4.17,4.5)$ & $(3.67,4.67,5)$ \\
Smart phone & $(3.67,4.67,5)$ & $(3.67,4.67,5)$ & $(3.17,4.17,4.5)$ & $(3.67,4.67,5)$ & $(3.67,4.67,5)$ \\
Smart motion sensor & $(2.33,3.33,4.00)$ & $(0,1,2)$ & $(2,3,4)$ & $(2.33,3.33,4.00)$ & $(3.67,4.67,5)$ \\
Smart smoke alarm & $(2.33,3.33,4.00)$ & $(0,1,2)$ & $(3.67,4.67,5)$ & $(2.33,3.33,4.00)$ & $(3.67,4.67,5)$ \\
Smart body analyzer & $(2,2.67,3.67)$ & $(2,2.67,3.67)$ & $(2.33,3.33,4.00)$ & $(2,2.67,3.67)$ & $(3.17,4.17,4.5)$ \\
Smart connected vehicle & $(2.67,3.67,4)$ & $(3.33,4.33,5)$ & $(2,2.67,3.67)$ & $(2,2.67,3.67)$ & $(2.33,3.33,4.00)$ \\
Smart toilet & $(2,2.67,3.67)$ & $(0,1,2)$ & $(2.33,3.33,4.00)$ & $(0,1,2)$ & $(2.33,3.33,4.00)$ \\
Smart wig & $(2.67,3.33,3.67)$ & $(0,1,2)$ & $(3.17,4.17,4.5)$ & $(0,1,2)$ & $(3.17,4.17,4.5)$ \\
Smart mobile service & $(3.67,4.67,5)$ & $(3.67,4.67,5)$ & $(3.67,4.67,5)$ & $(3.67,4.67,5)$ & $(3.33,4.33,5)$ \\
Smart defense technology & $(2,2.67,3.67)$ & $(0,1,2)$ & $(3.17,4.17,4.5)$ & $(2.83,3.83,4.5)$ & $(2.33,3.33,4.00)$ \\
Smart wheelchair & $(3.17,4.17,4.5)$ & $(0.5,0.83,1.83)$ & $(2,2.67,3.67)$ & $(0,1,2)$ & $(2,2.67,3.67)$ \\
\hline
\end{tabular}

result. The value of $\alpha$ ranged from $0,0.1, \ldots$, to 1 , so the maximal eigenvalue and eigenvector needed to be derived $1+10 \cdot 2^{C_{2}^{5}}=10,241$ times. The ACO method was implemented using MATLAB 2017 on a PC with i7-7700 CPU $3.6 \mathrm{GHz}$ and $8 \mathrm{~GB}$ RAM. The execution time was less than $11 \mathrm{~s}$. The results are summarized in Figs. 2 and 3. Among the result, $\tilde{w}_{3}$ and $\tilde{w}_{5}$ could not be very well approximated with TFNs, as illustrated in Fig. 4.

Thirteen smart technologies, shown in Table 3, were considered. The performances of smart technologies along five dimensions were evaluated by the same expert group using the following linguistic terms [16]:

Very poor: $(0,0,1)$

Poor: $(0,1,2)$

Moderate: $(1.5,2.5,3.5)$

Good: $(3,4,5)$

Very good: $(4,5,5)$

The evaluations by experts were averaged. The results are summarized in Table 3.

FWA was then applied to evaluate the sustainability of each smart technology application to mobile health care. The results are summarized in Fig. 5.

Subsequently, COG was applied to defuzzify the fuzzy sustainability of each smart technology application to mobile health care. The results are summarized in Table 4.

According to the experimental results,

1. Among thirteen smart technology applications to mobile health care, the application of smart mobile service achieved the highest sustainability, while the application of smart clothes was considered the least sustainable.

2. The ranking result is shown in Fig. 6. By setting a threshold of three for the derived sustainability, only five smart technology applications to mobile health care, including smart mobile service, smart phone, smart watch, smart connected vehicle, and smart smoke alarm, were sufficiently sustainable.

3. Two existing methods, FGMi-FWA and FEAi-WA, were also applied in the experiment for a comparison. The weights estimated using FEAi were crisp. Therefore, WA, instead of FWA, was subsequently applied. The results obtained using the two existing methods are compared with that obtained using the proposed methodology in Fig. 7. Although the results looked similar, there were significant differences between those of some smart technology applications to mobile health care, e.g., smart clothes, smart mobile service, and smart phone.

4. The ranking results using various methods are not the same, as illustrated by Table 5, showing that approximation methods such as FGMi and FEAi were subject to inaccuracy that might mislead the decision-making process.

5. The fuzzy consistency index is shown in Fig. 8, revealing that there was some inconsistency among experts' judgments. In addition, the range of the fuzzy consistency index was a bit wide, showing that there was considerable uncertainty behind the fuzzy consistency index. It was not strange since smart technology applications to mobile health care were still in their infancy.

6. To ascertain whether the advantage of the FGM-ACOFWA approach over the FGMi method in improving the 

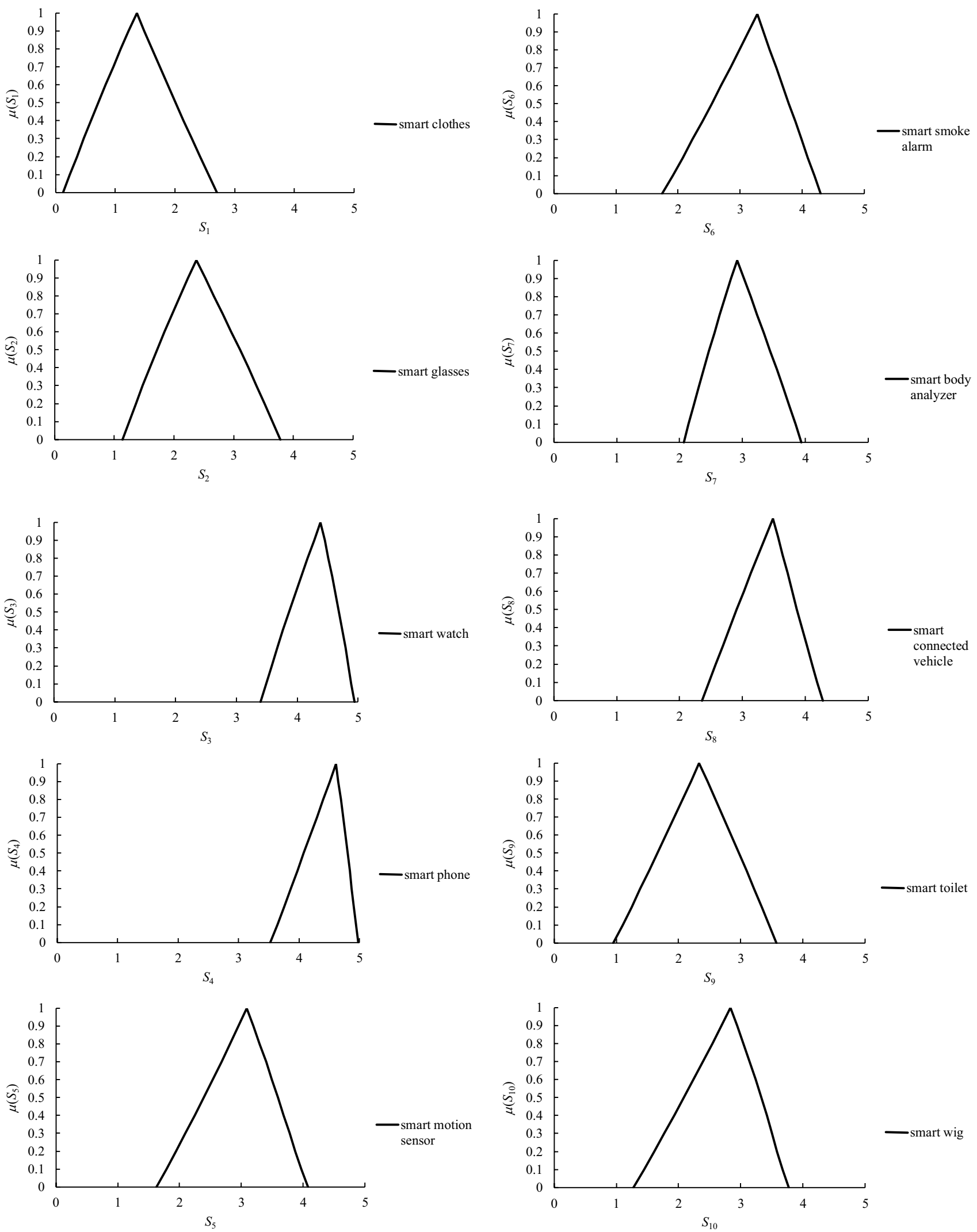

Fig. 5 The sustainability of each smart technology application to mobile health care 

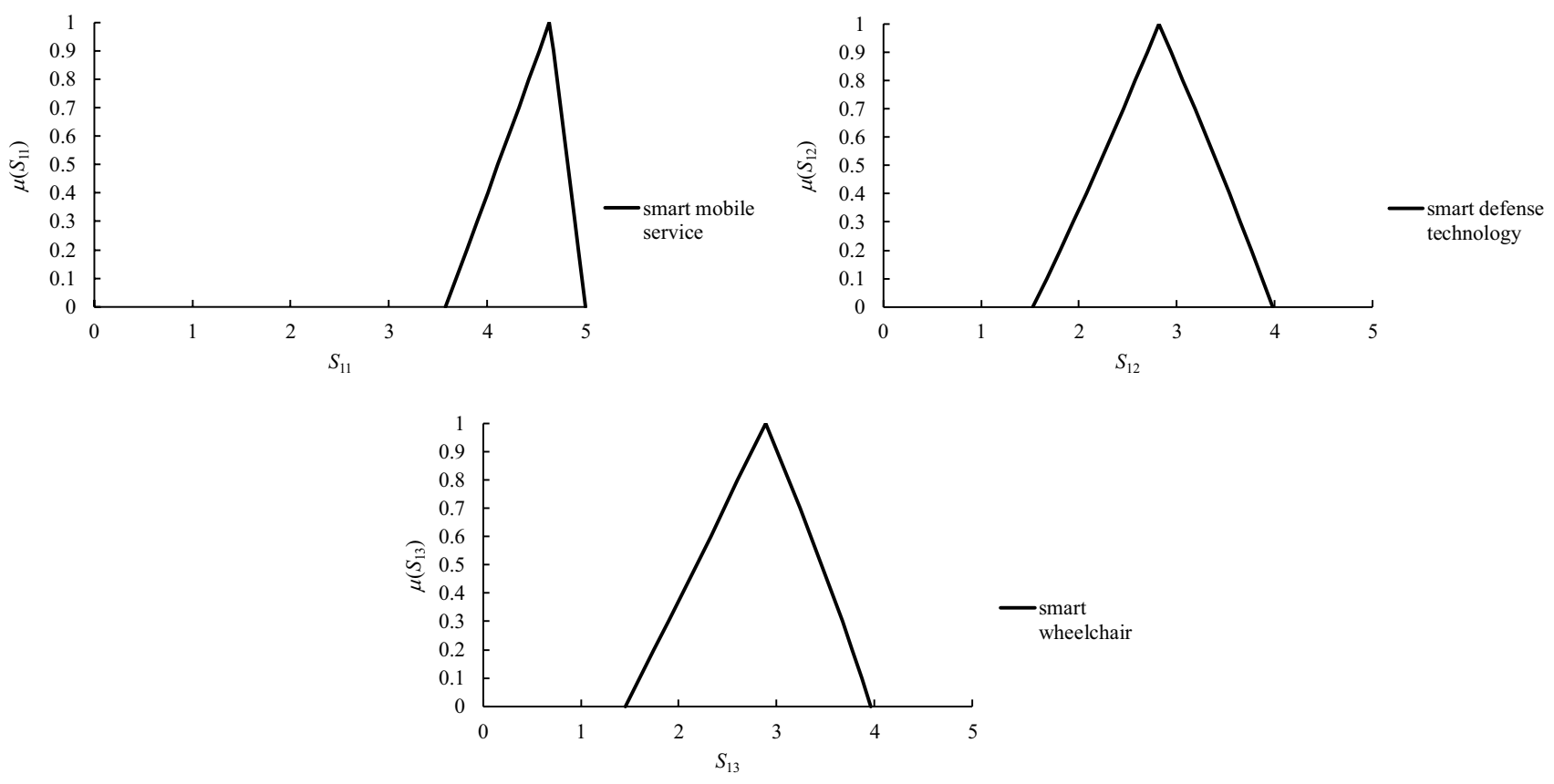

Fig. 5 (continued)

precision of deriving fuzzy weights was significant, the paired $t$ test was conducted:

$H_{0}$ : In improving the precision in terms of the average range of fuzzy weights, the performance of the FGMACO-FWA approach is the same as that of the FGMi method.

$H_{1}$ : In improving the precision in terms of the average range of fuzzy weights, the performance of the FGM-

Table 4 The defuzzification results

\begin{tabular}{ll}
\hline Smart technology & $\begin{array}{l}\text { Defuzzified } \\
\text { sustainability }\end{array}$ \\
\hline Smart clothes & 1.36 \\
Smart glasses & 2.40 \\
Smart watch & 4.31 \\
Smart phone & 4.50 \\
Smart motion sensor & 3.03 \\
Smart smoke alarm & 3.20 \\
Smart body analyzer & 2.94 \\
Smart connected vehicle & 3.42 \\
Smart toilet & 2.32 \\
Smart wig & 2.75 \\
Smart mobile service & 4.52 \\
Smart defense technology & 2.81 \\
Smart wheelchair & 2.84 \\
\hline
\end{tabular}

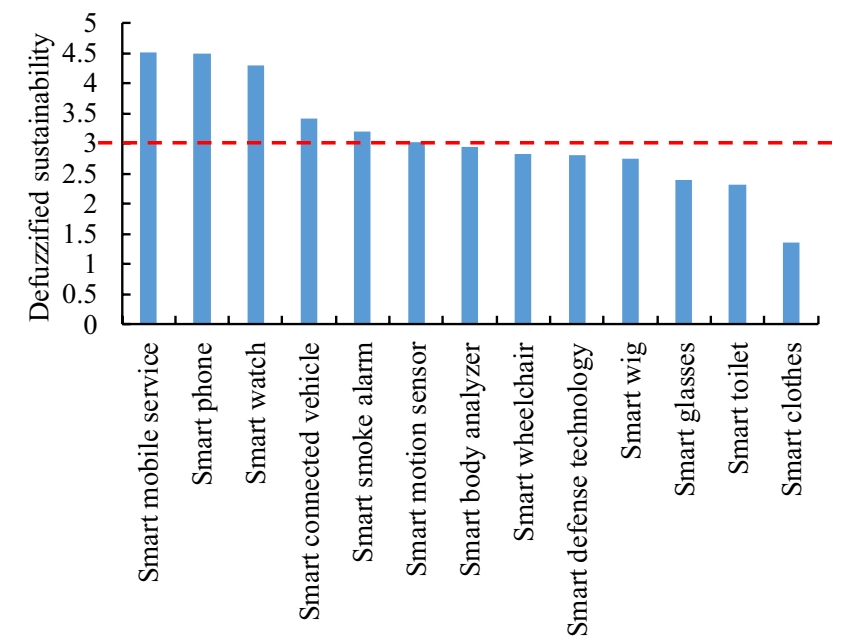

Fig. 6 The ranked results

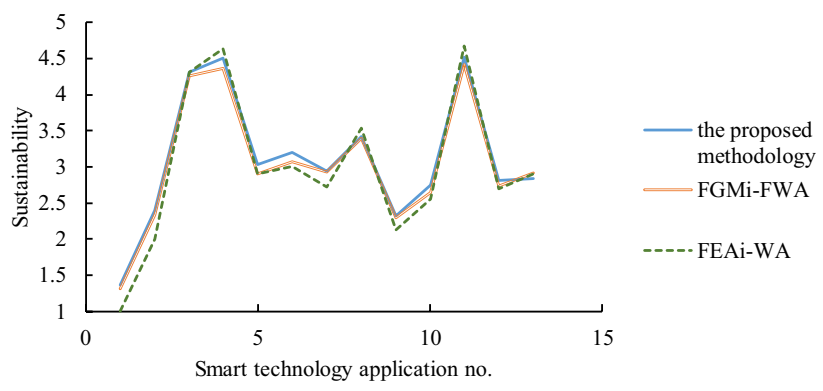

Fig. 7 Comparison of the results obtained using various methods 
Table 5 The ranking results using various methods

\begin{tabular}{llll}
\hline Rank & The proposed methodology & FGMi-FWA & FEAi-WA \\
\hline 1 & Smart mobile service & Smart mobile service & Smart mobile service \\
2 & Smart phone & Smart phone & Smart phone \\
3 & Smart watch & Smart watch & Smart watch \\
4 & Smart connected vehicle & Smart connected vehicle & Smart connected vehicle \\
5 & Smart smoke alarm & Smart smoke alarm & Smart smoke alarm \\
6 & Smart motion sensor & Smart body analyzer & Smart wheelchair \\
7 & Smart body analyzer & Smart wheelchair & Smart motion sensor \\
8 & Smart wheelchair & Smart motion sensor & Smart body analyzer \\
9 & Smart defense technology & Smart defense technology & Smart defense technology \\
10 & Smart wig & Smart wig & Smart wig \\
11 & Smart glasses & Smart glasses & Smart toilet \\
12 & Smart toilet & Smart toilet & Smart glasses \\
13 & Smart clothes & Smart clothes & Smart clothes \\
\hline
\end{tabular}

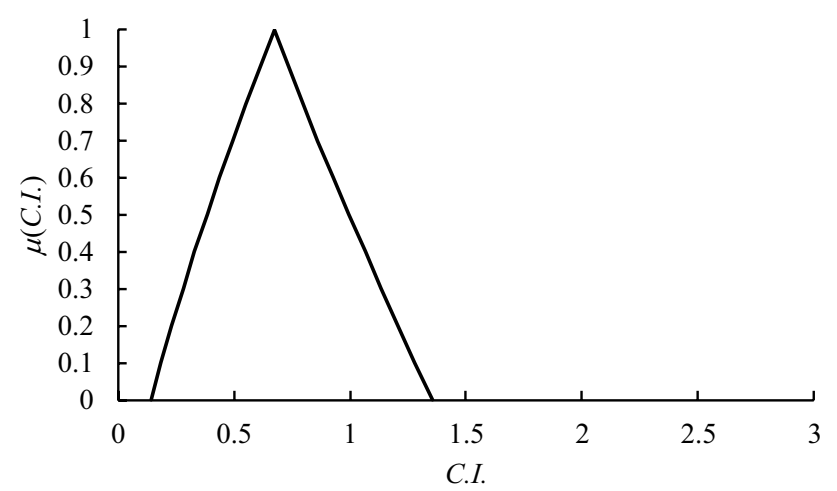

Fig. 8 The fuzzy consistency index

ACO-FWA approach is higher than that of the FGMi method.

Table 6 presents a summary of the results. The null hypothesis $\mathrm{H}_{0}$ was rejected at $\alpha=0.15$, indicating that the FGM-ACO-FWA approach was statistically superior to the FGMi method in improving the precision of deriving fuzzy weights.

7. The case of selecting contaminated site remedial countermeasures [33] was adopted to further elaborate the effectiveness of the proposed methodology. Four remedial countermeasures, environmental effectiveness, financial affordability, implementability, and social acceptability, were considered. There were two scenarios that were aggregated using FGM into the following fuzzy judgement matrix:

$$
\tilde{\mathbf{A}}=\left[\begin{array}{llll}
(1,1,1) & (0.41,0.87,1.58) & (0.45,0.82,2.00) & (1,2,4) \\
(0.63,1.15,2.45) & (1,1,1) & (0.50,1.00,2.00) & (0.45,0.82,2.00) \\
(0.50,1.22,2.24) & (0.50,1.00,2.00) & (1,1,1) & (0.45,0.82,2.00) \\
(0.20,0.50,1.00) & (0.50,1.22,2.24) & (0.50,1.22,2.24) & (1,1,1)
\end{array}\right]
$$

Table 6 Results of paired $t$ test

\begin{tabular}{lll}
\hline & FGMi & $\begin{array}{l}\text { FGM- } \\
\text { ACO- } \\
\text { FWA }\end{array}$ \\
\hline Mean & 0.29 & 0.19 \\
Variation & 0.07 & 0.01 \\
Observations & 5 & 5 \\
Pearson correlation coefficient & 0.99 & \\
Degree of freedom & 4 & \\
$t$ statistics & 1.14 & \\
$\mathrm{P}(T \leq t)$ one-tail & 0.15 & \\
$t$ critical one-tail & 2.13 & \\
$\mathrm{P}(T \leq t)$ two-tail & 0.32 & \\
$t$ critical two-tail & 2.78 & \\
\hline
\end{tabular}

which was processed using ACO. The derived fuzzy maximal eigenvalue and fuzzy weights are shown in Figs. 9 and 10. Obviously, the proposed methodology achieved a very high precision, especially in deriving $\tilde{\lambda}_{\max }$ and $\tilde{w}_{3}$. In contrast, existing approaches that approximated the results with TFNs would be imprecise. 


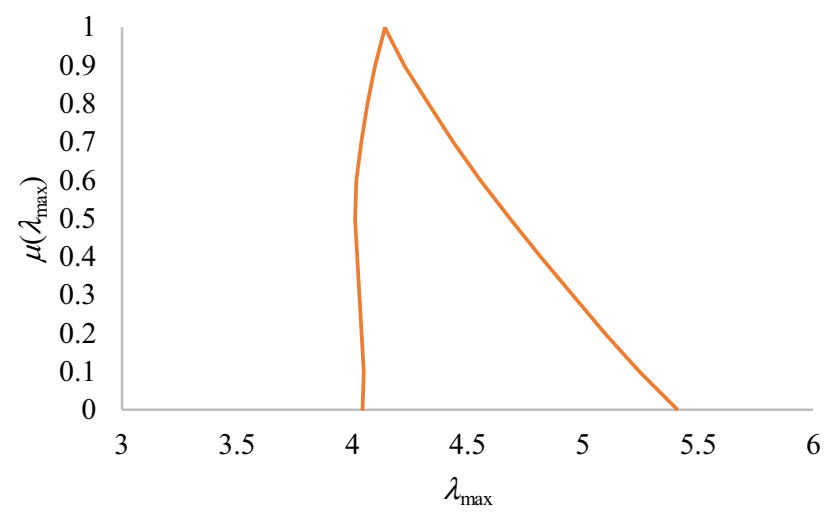

Fig. 9 The derived fuzzy maximal eigenvalue for another case

\section{Conclusions}

Smart technologies have been widely applied to mobile health care. However, existing applications of smart technologies to mobile health care face several difficulties. For example, there is a shortage of workforce for providing health care services, even in high-income countries. In addition, some smart technologies are obtrusive, which discourages mobile users from applying them. Further, aggregating heterogeneous data from multiple sources is a challenging task. As a result, some smart technology applications to mobile health care may not be sustainable. It is also a critical task to evaluate the sustainability of a smart technology application to mobile health care. To address these issues, the FGM-ACO-FWA approach is proposed in this study. In the proposed methodology, FGM is first applied to aggregate multiple experts' opinions on the relative importance of a factor critical to the sustainability of a smart technology application to mobile health care. Subsequently, ACO is applied to derive the absolute fuzzy importance level of each critical factor. Finally, FWA is applied to assess the sustainability of a smart technology application to mobile health care.

After applying the FGM-ACO-FWA approach to a real case, the following conclusions were drawn:

1. Five factors critical to the applications of smart technologies to mobile health care were identified as unobtrusiveness, supporting online social networking, relaxation of the related medical laws, the size of the health care market, and the correct identification of a user's need and situation.

2. Among thirteen smart technology applications to mobile health care, the most and least sustainable applications were smart mobile service and smart clothes, respectively.
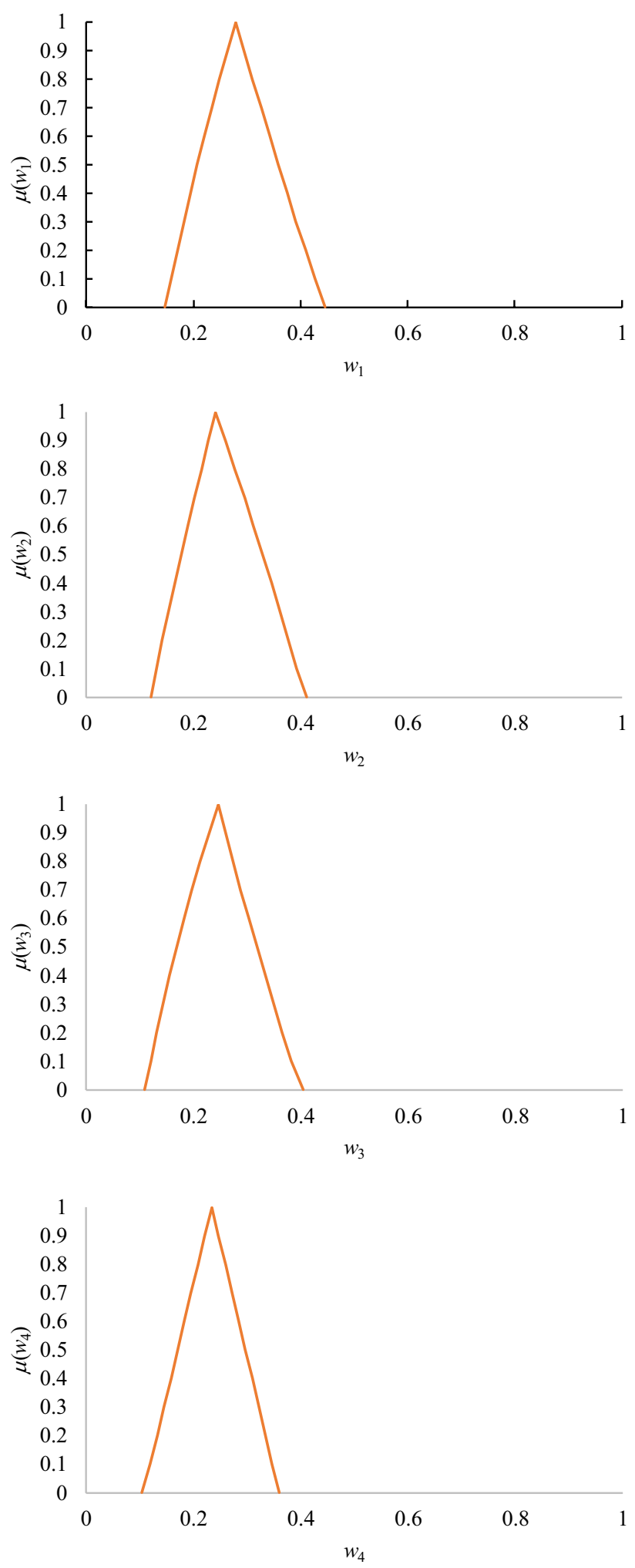

Fig. 10 The derived fuzzy weights for another case

3. The ranking result using the proposed methodology was somewhat different from those using two existing methods based on approximation. 
There are other ways to evaluate the sustainability of a smart technology application to mobile health care that can be explored in future studies to confirm the conclusions drawn in this study. In addition, experts' pairwise comparison results were aggregated before deriving fuzzy weights in this study, which assumed that experts could reach a consensus on the derived fuzzy weights. In a future study, fuzzy weights can be derived based on each expert's pairwise comparison result. Then, whether the fuzzy weights derived for all experts overlap implies the existence of a consensus. Further, the efficiency of the proposed methodology can be enhanced with computational intelligence techniques $[8,30]$. These constitute some directions for future research.

\section{Compliance with ethical standards}

Conflict of interest The author declare no conflict of interest.

Open Access This article is distributed under the terms of the Creative Commons Attribution 4.0 International License (http://creativeco mmons.org/licenses/by/4.0/), which permits unrestricted use, distribution, and reproduction in any medium, provided you give appropriate credit to the original author(s) and the source, provide a link to the Creative Commons license, and indicate if changes were made.

\section{References}

1. Ak MF, Gul M (2019) AHP-TOPSIS integration extended with pythagorean fuzzy sets for information security risk analysis. Complex Intell Syst 5(2):113-126

2. Amin MB, Banos O, Khan WA, Muhammad Bilal HS, Gong J, Bui D, Cho SH, Hussain S, Ali T, Akhtar U, Chung TC, Lee S (2016) On curating multimodal sensory data for health and wellness platforms. Sensors 16(7):980

3. Android Developers (2017) Sensors overview. https://devel oper.android.com/guide/topics/sensors/sensors_overview.html. Accessed 27 Jan 2019

4. Axisa F, Schmitt PM, Gehin C, Delhomme G, McAdams E, Dittmar A (2005) Flexible technologies and smart clothing for citizen medicine, home healthcare, and disease prevention. IEEE Trans Inf Technol Biomed 9(3):325-336

5. Ayağ Z, Samanlioglu F (2019) Fuzzy AHP-GRA approach to evaluating energy sources: a case of Turkey. Int J Energy Sector Manag. https://doi.org/10.1108/IJESM-09-2018-0012

6. Aydın S, Kahraman C (2019) Evaluation of firms applying to malcolm baldrige national quality award: a modified fuzzy AHP method. Complex Intell Syst 5(1):53-63

7. Bieber G, Kirste T, Urban B (2012) Ambient interaction by smart watches. In: Proceedings of the 5th international conference on pervasive technologies related to assistive environments, pp 391-396

8. Castillo O, Neyoy H, Soria J, Melin P, Valdez F (2015) A new approach for dynamic fuzzy logic parameter tuning in ant colony optimization and its application in fuzzy control of a mobile robot. Appl Soft Comput 28:150-159

9. Chen K, Chen JV, Yen DC (2011) Dimensions of self-efficacy in the study of smart phone acceptance. Comput Stand Interfaces 33(4):422-431
10. Chen JV, Yen DC, Chen K (2009) The acceptance and diffusion of the innovative smart phone use: a case study of a delivery service company in logistics. Inf Manag 46(4):241-248

11. Chen T (2015) Enhancing the performance of a ubiquitous location-aware service system using a fuzzy collaborative problem solving strategy. Comput Ind Eng 87:296-307

12. Chen $T$ (2016) Ubiquitous multicriteria clinic recommendation system. J Med Syst 40:113

13. Chen $\mathrm{T}$ (2017) Ubiquitous clinic recommendation by predicting a patient's preferences. In: Electronic commerce research and applications, 23, May-June, 14-23

14. Chen T (2019) Guaranteed-consensus posterior-aggregation fuzzy analytic hierarchy process method. Neural Comput Appl. https:// doi.org/10.1007/s00521-019-04211-y

15. Chen T, Chiu M-C (2018) Smart technologies for assisting the life quality of persons in a mobile environment - a review. J Ambient Intell Hum Comput 9(2):319-328

16. Chen TY, Ku TC, Tsui CW (2008) Determining attribute importance based on triangular and trapezoidal fuzzy numbers in fuzzy measures. In: the 19th international conference on multiple criteria decision making, pp 75-76

17. Chipara O, Lu C, Bailey TC, Roman GC (2010) Reliable clinical monitoring using wireless sensor networks: experiences in a stepdown hospital unit. In: Proceedings of the 8th ACM conference on embedded networked sensor systems, pp 155-168

18. Chiu M-C, Chen T (2019) Assessing sustainable effectiveness of the adjustment mechanism of a ubiquitous clinic recommendation system. In: Health care management science (in press)

19. Curtis DW, Pino EJ, Bailey JM, Shih EI, Waterman J, Vinterbo SA, Stair TO, Guttag JV, Greenes RA, Ohno-Machado L (2008) SMART - an integrated wireless system for monitoring unattended patients. J Am Med Inf Assoc 15(1):44-53

20. Demiris G, Hensel BK, Skubic M, Rantz M (2008) Senior residents' perceived need of and preferences for "smart home" sensor technologies. Int J Technol Assess Health Care 24(1):120-124

21. Demirkan H (2013) A smart healthcare systems framework. It Professional 15(5):38-45

22. Free C, Phillips G, Galli L, Watson L, Felix L, Edwards P, Patel V, Haines A (2013) The effectiveness of mobile-health technologybased health behaviour change or disease management interventions for health care consumers: a systematic review. PLoS Med 10(1):e1001362

23. Gaul S, Ziefle M (2009) Smart home technologies: Insights into generation-specific acceptance motives. In: Symposium of the Austrian HCI and usability engineering group, pp 312-332

24. Güran A, Uysal M, Ekinci Y, Güran CB (2017) An additive FAHP based sentence score function for text summarization. Inf Technol Control 46(1):53-69

25. Hamel MB, Cortez NG, Cohen IG, Kesselheim AS (2014) FDA regulation of mobile health technologies. N Engl J Med 371(4):372

26. Isken MW, Sugumaran V, Ward TJ, Minds D, Ferris W (2005) Collection and preparation of sensor network data to support modeling and analysis of outpatient clinics. Health Care Manag Sci $8(2): 87-99$

27. Jordan M (2011) What is 'smart' technology? http://knowit.co. nz/2011/08/what-is-smart-technology. Accessed 27 Jan 2019

28. Kim KJ, Shin DH (2015) An acceptance model for smart watches: implications for the adoption of future wearable technology. Int Res 25(4):527-541

29. Lima Junior FR, Osiro L, Carpinetti LCR (2014) A comparison between fuzzy AHP and Fuzzy TOPSIS methods to supplier selection. Appl Soft Comput 21:194-209

30. Olivas F, Valdez F, Castillo O, Gonzalez CI, Martinez G, Melin P (2017) Ant colony optimization with dynamic parameter 
adaptation based on interval type-2 fuzzy logic systems. Appl Soft Comput 53:74-87

31. Peter J (2018) Achieving scale, sustainability and impact: a donor perspective on a mobile health messaging service and help desk (MomConnect) for South African mothers. BMJ Glob Health 3(Suppl 2):e000562

32. Porzi L, Messelodi S, Modena CM, Ricci E (2013) A smart watchbased gesture recognition system for assisting people with visual impairments. In: Proceedings of the 3rd ACM international workshop on interactive multimedia on mobile and portable devices, pp 19-24

33. Promentilla MAB, Furuichi T, Ishii K, Tanikawa N (2008) A fuzzy analytic network process for multi-criteria evaluation of contaminated site remedial countermeasures. J Environ Manag 88(3):479-495

34. Rathod R (2012) Sensors used in smartphone. http://myphonefac tor.in/2012/04/sensors-used-in-a-smartphone/. Accessed 26 Jan 2019

35. Rawassizadeh R, Price BA, Petre M (2015) Wearables: has the age of smartwatches finally arrived? Commun ACM 58(1):45-47

36. Sarasohn-Kahn J (2010) How smartphones are changing health care for consumers and providers. http://www.chcf.org/publicatio ns/2010/04/how-smartphones-are-changing-health-care-for-consu mers-and-providers. Accessed 25 Jan 2019

37. Saaty TL (1980) The analytic hierarchy process. McGraw-Hill, New York

38. Shcherbina A, Mattsson CM, Waggott D, Salisbury H, Christle JW, Hastie T, Wheeler MT, Ashley EA (2017) Accuracy in wrist-worn, sensor-based measurements of heart rate and energy expenditure in a diverse cohort. J Pers Med 7(2):3

39. Singh S, Olugu EU, Musa SN, Mahat AB (2018) Fuzzy-based sustainability evaluation method for manufacturing SMEs using balanced scorecard framework. J Intell Manuf 29(1):1-18
40. Sirisawat P, Kiatcharoenpol T (2018) Fuzzy AHP-TOPSIS approaches to prioritizing solutions for reverse logistics barriers. Comput Ind Eng 117:303-318

41. Sixsmith A, Johnson N (2004) A smart sensor to detect the falls of the elderly. IEEE Pervasive Comput 3(2):42-47

42. SmartWatches.org (2017) The 5 best smartwatches for health and fitness. http://smartwatches.org/learn/the-5-best-smartwatch es-for-health-and-fitness/. Accessed 26 Jan 2019

43. Tsai H-R, Chen T (2014) Enhancing the sustainability of a location-aware service through optimization. Sustainability 6:9441-9455

44. van Doorn M (2015) What does SMART technology actually mean? http://labs.sogeti.com/wat-smart-technology-actuallymean/. Accessed 27 Jan 2019

45. Van Broekhoven E, De Baets B (2006) Fast and accurate center of gravity defuzzification of fuzzy system outputs defined on trapezoidal fuzzy partitions. Fuzzy Sets Syst 157(7):904-918

46. Ventola CL (2014) Mobile devices and apps for health care professionals: uses and benefits. Pharm Therap 39(5):356

47. Vodopivec-Jamsek V, de Jongh T, Gurol-Urganci I, Atun R, Car J (2008) Mobile phone messaging for preventive health care. Cochrane Database Syst Rev 12:CD007457. https://doi. org/10.1002/14651858.CD007457.pub2

48. Wang Y-C, Chen T (2019) A partial-consensus posterior-aggregation FAHP method-supplier selection problem as an example. Mathematics 7(2): 179

Publisher's Note Springer Nature remains neutral with regard to jurisdictional claims in published maps and institutional affiliations. 